\title{
Radionuclide Imaging of Infective Endocarditis: State of Art and Future Perspective
}

\author{
Stella Marchetta ${ }^{1}$ - Nadia Withofs ${ }^{2} \cdot$ Paola Anna Erba ${ }^{3}$ - Gilbert Habib ${ }^{4,5}$. \\ Roland Hustinx ${ }^{2}$ • Patrizio Lancellotti ${ }^{1,6,7}$
}

(C) Springer Science+Business Media, LLC 2017

\begin{abstract}
Purpose of Review Infectious endocarditis is a serious disease requiring rapid diagnosis and accurate risk stratification to offer the best therapeutic strategy. Infection of prosthetic valve (PV) and cardiovascular implantable electronic device (CIED) is increasing due to the ageing of the population and the growing number of implants. Foreign material infection remains clinically challenging given the limitation of ultrasound techniques in this context whereas the diagnosis must be precocious.

Recent Findings ${ }^{18} \mathrm{~F}$-fluorodeoxyglucose positron emission tomography/computed tomography $\left(\left[{ }^{18} \mathrm{~F}\right] \mathrm{FDG}\right.$ PET/CT $)$ and radiolabelled leukocytes single-photon emission computed tomography/computed tomography (SPECT/CT) are commonly used for this purpose.
\end{abstract}

This article is part of the Topical Collection on Cardiac Nuclear Imaging

Patrizio Lancellotti

plancellotti@chu.ulg.ac.be

1 University of Liège hospital, GIGA Cardiovascular Science, Heart Valve Clinic, Imaging Cardiology, CHU Sart tilman, Liège, Belgium

2 Nuclear Medicine and Oncological Imaging Division, Medical Physics Department, CHU Sart tilman, Liège, Belgium

3 Nuclear Medicine, Department of Translational Research and New Technology in Medicine, University of Pisa, Pisa, Italy

4 URMITE, Aix Marseille Université, UM63, CNRS 7278, IRD 198, INSERM 1095, IHU, Méditerranée Infection, Marseille, France

5 APHM, La Timone Hospital, Cardiology Department, Marseille, France

6 Gruppo Villa Maria Care and Research, Anthea Hospital, Bari, Italy

7 Department of Cardiology, University Hospital Sart Tilman, B 4000 Liege, Belgium
Summary In the present article, we summarized the available evidence for the use of nuclear imaging for the evaluation of infectious endocarditis.

Keywords Infective endocarditis - Cardiac prosthetic valves . Cardiovascular implantable electronic device $\cdot\left[{ }^{18} \mathrm{~F}\right] \mathrm{FDG}$ positron emission tomography $\cdot$ Radiolabelled leukocyte scintigraphy $\cdot$ Embolic events

\section{Introduction}

Infective endocarditis (IE) remains a deadly disease despite continuous advances in medical and surgical management $[1-3,4 \cdot 0]$. In the recent years, epidemiology of IE has undergone significant changes with increasing number of elderly patients presenting with degenerative valvular disease, nosocomial infections, or device-related IE (prosthetic valves (PV), cardiac implantable electronic devices (CIED)). Though the diagnosis of IE remains challenging, the main advantage of timely diagnosis is the potential to prevent complications such as embolic events, septic complications, and valvular destruction [1]. In daily practice, the diagnosis of IE relies on the modified Duke criteria that use typical clinical signs and symptoms and positive blood cultures to reach a definitive diagnosis when the valve/device can be shown to be affected on echocardiography (vegetation, abscess or pseudoaneurysm, and new PV dehiscence). However, this technique has a sensitivity and specificity of approximately 80\% for native valve endocarditis (NVE) [2]. The diagnostic accuracy is even lower for PVE [3] or CIED infection [4••], in which echocardiography gives uncertain results in up to $30 \%$ of cases. The major drawbacks of transthoracic (TTE) and transoesophageal (TOE) echocardiography are related to patient morphology, instrumental settings, transducer position, operator skill, artefacts secondary to calcifications and reverberations of 
metallic structures, and the disease course (less accurate in the early stage) [5]. The shortcomings of the diagnosis of IE based on morphological changes have triggered an increasing use of functional imaging $\left({ }^{18} \mathrm{~F}\right.$-fluorodeoxyglucose $\left(\left[{ }^{18} \mathrm{~F}\right] \mathrm{FDG}\right)$ positron emission tomography (PET) and radiolabelled leukocytes single-photon emission computed tomography (SPECT)) for the evaluation of the metabolic activity caused by the infection, prior to any structural (morphological) change. When combined with standard diagnostic tests, functional imaging procedures have been shown to reduce the rate of misdiagnosed cases of IE. The recent European Society of Cardiology (ESC) guidelines have incorporated molecular imaging in the diagnostic work-up of PVE, whereas in case of CIED infection, the indication is less supported by the literature (recommendation class IIB level of evidence C) [1]. According to the new guidelines, the finding of abnormal $\left[{ }^{18} \mathrm{~F}\right] \mathrm{FDG}$ or radiolabelled leukocyte uptake around PV represents a major Duke criterion, whilst an embolic event or infectious aneurysms detected by imaging represents a minor criterion. All this has highlighted the major role of the imaging specialists as part of the 'Endocarditis Team', in the management of IE. In the present article, we summarized the available evidence for the use of nuclear imaging for the evaluation of IE.

\section{$\left[{ }^{18}\right.$ F $]$ FDG PET/CT and Radiolabelled White Blood Cell (WBC) SPECT/CT}

$\left[{ }^{18} \mathrm{~F}\right] \mathrm{FDG} \mathrm{PET} / \mathrm{CT}$ is a non-invasive imaging technique evaluating the metabolic activities of healthy and pathological tissues. $\left[{ }^{18} \mathrm{~F}\right] \mathrm{FDG}$ is a glucose analogue, which is incorporated by cells with enhanced expression of the glucose transporters (GLUT 1 and GLUT 3), as in presence of activated leukocytes, macrophages and $\mathrm{CD}^{+}$ lymphocytes. Once in the cytoplasm, $\left[{ }^{18} \mathrm{~F}\right] \mathrm{FDG}$ is phosphorylated and trapped into the cells [6••]. By the combination of $\left[{ }^{18} \mathrm{~F}\right]$ FDG PET and high-resolution computed tomography $(\mathrm{CT})$, the functional information and the anatomical details are entailed in single image [7]. Wholebody PET/CT is performed using a single acquisition time-point, generally 45-60 min after intravenous injection of $\left[{ }^{18} \mathrm{~F}\right] \mathrm{FDG}$, with an emission time/bed position depending on the sensitivity of the scanner. The field of acquisition is usually derived from oncology studies from skull base to mid thighs. The majority of PET/CT studies consists of a protocol comprising a scanogram/scout scan/topogram and CT-AC. The simultaneous acquisition of a standard diagnostic $\mathrm{CT}$ scan with intravenous contrast agent is possible. PET images are visually evaluated to search for area of increased $\left[{ }^{18} \mathrm{~F}\right] \mathrm{FDG}$ uptake, taking into consideration the pattern (focal, linear, diffuse), intensity, and relationship to areas of physiologic distribution. PET information is compared with morphologic information obtained by CT. Semi-quantitative analysis by maximal standardized uptake value (SUVmax) or other semi- quantitative parameters is also possible. However, SUV has not been validated in infection $\left[8^{\bullet}\right]$. When $\left[{ }^{18} \mathrm{~F}\right] \mathrm{FDG}$ $\mathrm{PET} / \mathrm{CT}$ is used to diagnose cardiac and pericardiac infection, patient preparation becomes very important due to the possible presence of physiologic uptake of $\left[{ }^{18} \mathrm{~F}\right] \mathrm{FDG}$ in normal myocardium (for details about this topic, see the Addendum). The current SNMMI/ASNC/SCCT guidelines recommend preparation with a fat-enriched diet lacking carbohydrates for $12-24 \mathrm{~h}$ prior to the scan, a 12 $18 \mathrm{~h}$ fast, and/or the use of intravenous heparin approximately 15 min prior to $\left[{ }^{18} \mathrm{~F}\right] \mathrm{FDG}$ injection [9]. To prevent misinterpretation of a positive scan due to early imaging after valve implantation, the ESC Guidelines recommend not to consider $\left[{ }^{18} \mathrm{~F}\right]$ FDG PET results in the 3-month period following prosthetic heart valve (PHV) implantation [5] (Table 1).

White blood cell (WBC) SPECT/CT imaging uses autologous radiolabelled leukocytes after radiolabelling with ${ }^{111} \mathrm{In}$-oxine and ${ }^{99 \mathrm{~m}} \mathrm{Tc}$-hexamethylpropyleneamine oxime (HMPAO). Imaging acquisition protocol includes planar acquisitions at $30 \mathrm{~min}$ (early images), 4-6 h (delayed images) and 20-24 h (late images) after reinjection of ${ }^{99 \mathrm{~m}} \mathrm{Tc}$ HMPAO $/{ }^{111}$ In-oxine-WBC. A SPECT/CT acquisition is mandatory as part of the standard imaging protocol, and it is usually acquired at $4-6 \mathrm{~h}$ and/or at $20-24 \mathrm{~h} \mathrm{p} . \mathrm{i} .{ }^{99 \mathrm{~m}} \mathrm{Tc}-$ HMPAO is preferred because more available, provides better image quality with lower patients radiation burden $[6,10 \cdot]$. The interpretation of WBC scintigraphy should always begin with a visual quality control performed on WB images and chest planar acquisitions. The signal kinetics between 4 and $6 \mathrm{~h}$ and $20-24 \mathrm{~h}$ acquisitions is an important feature for interpretation: any stable-increased uptake intensity or size over time, confirmed at SPECT/ $\mathrm{CT}$, is highly suggestive of infection (Table 1).

\section{Native Valve Endocarditis}

In native valve endocarditis (NVE), the usefulness of functional nuclear imaging has yet to be demonstrated. Indeed, few studies evaluated this specific clinical setting. In a recent prospective study concerning 72 patients with bacteraemia (Staphylococcus, Streptococcus species and Enterococcus species), Kouijzer et al. reported a limited accuracy (sensitivity $39 \%$, specificity was $93 \%$ ) of $\left[{ }^{18} \mathrm{~F}\right] \mathrm{FDG}$ PET/CT for diagnosing IE. Only two patients had PV in this study $[11 \bullet \cdot]$. Other smaller studies did not show better results [12••] (Table 2). Nevertheless, this technique may allow early detection of metastatic infectious disease with a high sensitivity $(87-100 \%)$ and specificity $(80 \%)$ [12••], at a reasonable cost-effectiveness, especially in patients with Grampositive bacteraemia [13•].

99m TC-HMPAO WBC SPECT/CT has been used by Erba et al. in a mixed population (16 NVE and 35 
Table 1 Comparison of $\left[{ }^{18} \mathrm{~F}\right] \mathrm{FDG}$ PET/CT and WBC SPECT/CT for the diagnosis of IE

\begin{tabular}{|c|c|c|}
\hline & Advantages & Disadvantages \\
\hline $\begin{array}{l}{\left[{ }^{18} \mathrm{~F}\right] \mathrm{FDG} \text { PET/CT }} \\
\text { "Sensitive imaging } \\
\text { technique" }\end{array}$ & $\begin{array}{l}\text { - Higher spatial resolution compared with SPECT } \\
\text { - Fast images acquisition ( } 80 \text { min with } 60 \text { min preparation } \\
\text { and } 20 \text { min scan time) } \\
\text { - Decreases cases of "possible IE" and rise-up so-called } \\
\text { "defined" endocarditis in PVE and CIED } \\
\text { - Helps in the diagnosis of deep, superficial or lead in } \\
\text { pacemaker's infection } \\
\text { - Seems to be promising in diagnosis LVAD related infection } \\
\text { - Detects paravalvular lesions \& extracardiac complications } \\
\text { (even silent events) } \\
\text { - Detects other sources of fever and/or neoplasia in a single } \\
\text { time } \\
\text { - Seems demonstrating valvular damage before ultrasounds } \\
\text { - When combined with CTA, provides high spatial } \\
\text { resolution images to identify structural damage and } \\
\text { analyse the coronary artery }\end{array}$ & $\begin{array}{l}\text { - Radiation exposure: effective dose } \sim 8 \mathrm{mSv} \text { ( } 4 \mathrm{mSv} \text { for an injected dose } \\
\text { of } 210 \mathrm{MBq}{ }^{18} \mathrm{~F}-\mathrm{FDG} \text { and } \sim 4 \mathrm{mSv} \text { for the low dose } \mathrm{CT}^{\mathrm{a}} \\
\text { - Poor accuracy with NVE } \\
\text { - Cannot differentiate infectious from non infectious inflammation and } \\
\text { not reliable within } 3 \text { months postoperatively for PVE and } 6 \text { weeks for } \\
\text { CIED } \\
\text { - No standardized protocols for preparation of patients and acquisition of } \\
\text { images - dietary restriction - test uninterpretable in areas with high } \\
\text { background activity such as heart when high myocardial uptake } \\
\text { - Artefacts with heartbeat and breathing } \\
\text { - Possible false-negatives test in patients with small vegetation }(<6 \mathrm{~mm}) \\
\text { and prolonged antibiotic therapy } \\
\text { - Possible false-positives in active thrombi, cardiac tumours or metastasis, } \\
\text { foreign body reactions, atrial fibrillation and lipomatous hypertrophy } \\
\text { - Not available in several centres \& costly }\end{array}$ \\
\hline $\begin{array}{l}\text { WBC SPECT/CT } \\
\text { "Specific imaging } \\
\text { technique" }\end{array}$ & $\begin{array}{l}\text { - High positive predictive value and specificity for acute } \\
\text { infection: useful in direct post-operative } \\
\text { - Helps to detect abscesses and paravalvular infections } \\
\text { - Helps for detecting septic embolism even in asymptomatic } \\
\text { patients } \\
\text { - No false-positives in cardiac infection } \\
\text { - Later SPECT images acquisition possible with }{ }^{111} \text { In-oxine } \\
\text { labelled leukocytes, valuable in low-grade infection }\end{array}$ & $\begin{array}{l}\text { - Radiation exposure higher effective dose with }{ }^{111} \text { In-oxine- labelled } \\
\text { WBC }(\sim 11 \mathrm{mSv}) \text { than with }{ }^{99 \mathrm{~m}} \mathrm{Tc}-\mathrm{HMPAO}-\text { labelled WBC }(\sim 3 \mathrm{mSv})^{\mathrm{a}} \\
\text { - Limited spatial resolution } \\
\text { - May require later images acquisition ( } 24 \mathrm{~h} \text { with four visits and } 2 \text { scans) } \\
\text { and multiple appointments } \\
\text { - Artefacts of metal } \\
\text { - Handling of blood products } \\
\text { - False-negatives with Enterococcus and Candida infections } \\
\text { (nonpyogenic microorganisms) } \\
\text { - Effect of antibiotherapy unclear } \\
\text { - False-positives and false-negatives regarding distant septic embolisms } \\
\text { - Requires special equipment and not available in several centres \& costly }\end{array}$ \\
\hline
\end{tabular}

${ }^{\text {a }}$ Effective dose calculated in an adult male using the "Nuclear Medicine Radiation Dose Tool" of the Society of nuclear medicine and molecular imaging available at http://www.snmmi.org/ClinicalPractice/doseTool.aspx?ItemNumber=11216\&navItemNumber=11218

PVE) showing that no cases were undiagnosed when either the echography or the blood cultures were positive [14••] (Table 2) (Fig. 1).

\section{Prosthetic Valve Endocarditis}

The rate of prosthetic valve endocarditis (PVE) ranges from 1-6 to $15 \%$, being higher in revision surgery [15]. Conversely to NVE, which is usually limited to the presence of a vegetation, PVE infection generally spread along the sewing ring and leads to peri-annular extension (pseudo-aneurysms, abscess) [16]. In a recent prospective study concerning 72 patients, Saby et al. showed that adding abnormal ${ }^{18}$ F-FDG uptake around a PV to the modified Duke criteria at admission increased the sensitivity for definite PVE from 70 to $97 \%$ (Table 2) [17••]. This result was due to a significant reduction in the number of possible PVE cases from 56 to $32 \%$. In addition, $\left[{ }^{18} \mathrm{~F}\right] \mathrm{FDG}$ PET/CT allowed detection of valvular damage before ultrasounds [8, 18]. Other smaller studies also confirmed these data [12]. More recently, Pizzi et al. reported the incremental value of $\left[{ }^{18} \mathrm{~F}\right] \mathrm{FDG}$ PET/ CT imaging in association with CT-angiography (CTA) over the modified Duke score at admission for the diagnosis of IE in 75 patients with $\mathrm{PV}$ or intra-cardiac devices. $\left[{ }^{18} \mathrm{~F}\right] \mathrm{FDG}$ PET/CTA offered excellent diagnostic performances (sensitivity 87\%, specificity $90 \%$ ) for the detection of IE. $\left[{ }^{18} \mathrm{~F}\right]$ FDG PET/CTA in association with Duke criteria allowed reclassifying 90\% (35/39) of cases initially classified as "possible" IE and provided a more conclusive diagnosis (definite/reject) in $95 \%$ (71/75) of cases (Table 2). Besides, $\left[{ }^{18} \mathrm{~F}\right]$ FDG PET/CTA identified a greater number of anatomic lesions than PET/CT or echocardiography, many of them relevant for clinical and surgical decision-making (pseudoaneurysms, fistulas, thrombosis and coronary involvement) [18].

A clear advantage of CTA in IE patients is the ability to assess the entire chest (identification of septic pulmonary infarcts and abscesses), the aorta and the coronary arteries before contemplated surgery [18••]. Several reports have also highlighted the potential added value of radiolabelled leukocytes SPECT/CT in the diagnosis of PVE (sensitivity 64-90\%, specificity 36-100\%), even in the early post-intervention phase [14, 19•]. As for $\left[{ }^{18} \mathrm{~F}\right]$ FDG PET/CT, ${ }^{99 \mathrm{~m}} \mathrm{Tc}-\mathrm{WBC}$ SPECT/CT has an excellent positive predictive value for the detection of perivalvular infection and abscesses in patients with a suspicion of PVE. In addition, the intensity of radiolabelled leucocyte accumulation in the perivalvular area detected with scintigraphy represents an interesting marker of local infectious activity and extension. Patients 


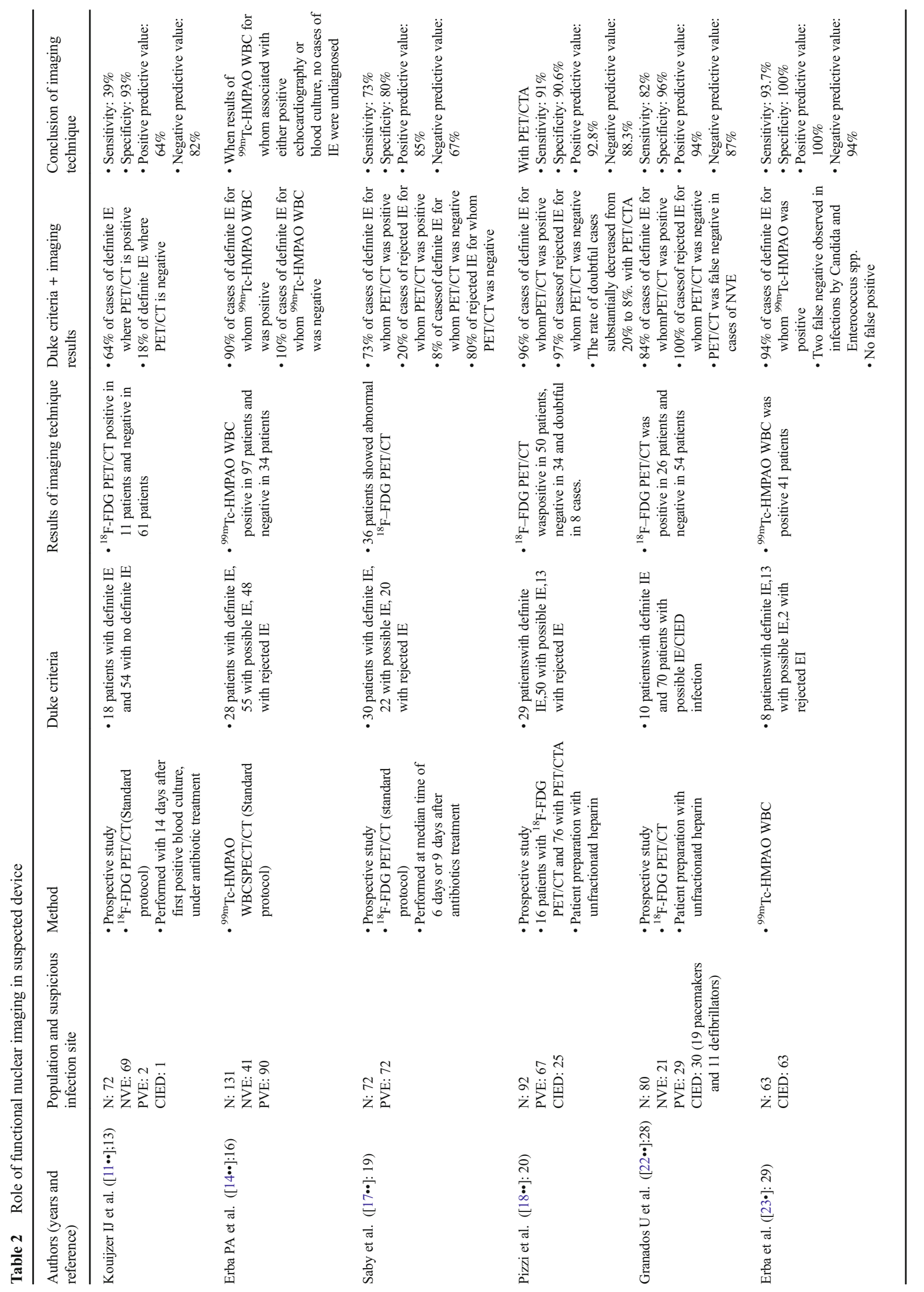



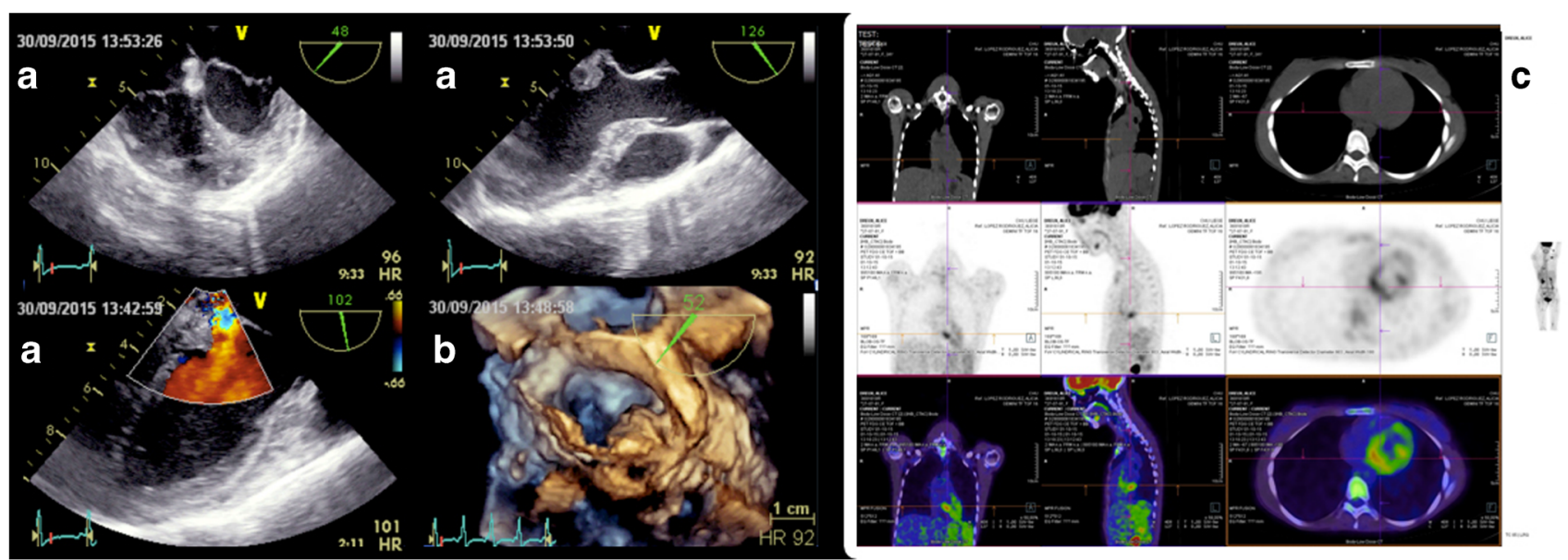

Fig. 1 To the left of the picture, transophagic ultrasound showing massive mitral endocarditis on native valve (a standard sections; b 3D sections). To the right of the picture (c), ${ }^{18} \mathrm{~F}-\mathrm{FDG}$ PET/CT images (from

left to right; coronal slice, sagittal slice, axial slice) of a patient with a native mitral valve endocarditis (red arrows, SUVmax: 4.4)

with a mild activity on the first exam disappearing on the second imaging evaluation seem to have a favourable outcome [19•] (Fig. 2).

\section{Cardiac Device-Related IE}

Cardiac device-related IE (CDRIE) - infection extending to the electrode leads, cardiac valve leaflets, or endocardial surfaceshould be distinguished from local device infection (pocket/generator). An incidence of 1.4 per 1000 device-years of definite CDRIE has been reported [3]. As for PV, ${ }^{99 \mathrm{~m}} \mathrm{Tc}-W B C$ SPECT/ $\mathrm{CT}$ and $\left[{ }^{18} \mathrm{~F}\right] \mathrm{FDG}$ PET/CT are able to differentiate CDRIE from post-implantation changes (i.e., pocket hematoma) and to characterize the extension of the infectious process (Fig. 3). $\left[{ }^{18} \mathrm{~F}\right] \mathrm{FDG} \mathrm{PET} / \mathrm{CT}$ diagnoses pocket infection with a good sensitivity $(87-91 \%)$ and specificity (93-100\%). The use of functional nuclear imaging can, therefore, allow the distinction between superficial and deep pocket infection, which necessitates removal of the generator rather than a medical treatment [21•]. The diagnostic accuracy of $\left[{ }^{18} \mathrm{~F}\right] \mathrm{FDG}$ PET/CT for lead infections is, however, lower (sensitivity $24-100 \%$, specificity $79-100 \%$ ) [12]. Such a finding is mainly related to the small size of the vegetations along the leads, which are often under the spatial resolution of the system $\left.[20] .{ }^{18} \mathrm{~F}\right] \mathrm{FDG}$ PET/CT in association with Duke criteria also allowed reclassifying most of cases initially classified as "possible" IE [18]. Erba et al. also reported a good diagnostic accuracy (94\%) of ${ }^{99 m}$ TC-HMPAO WBC SPECT/CT for CIED infection and for distinguishing infection

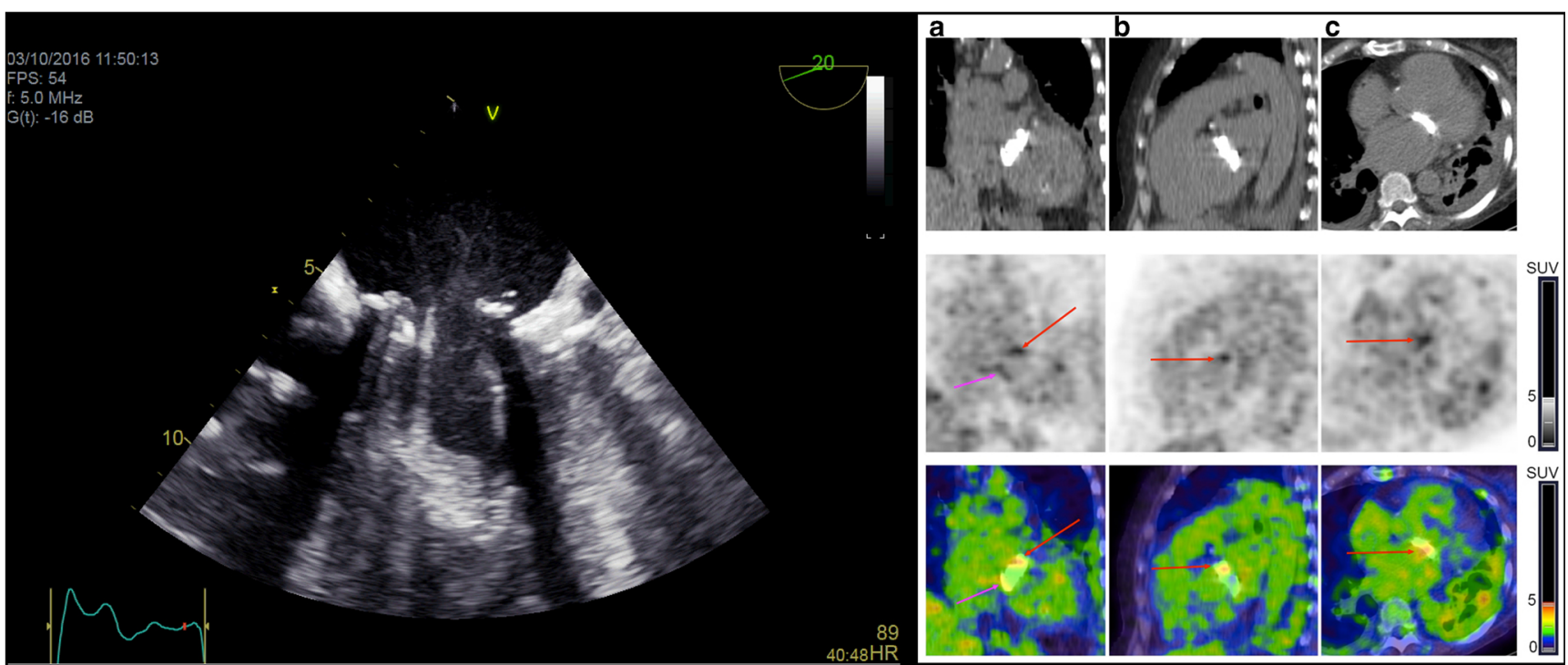

Fig. 2 To the left of the picture, transoephagic ultrasound of a mitral prosthesis revealing no obvious anomaly. To the right, $\left[{ }^{18} \mathrm{~F}\right] \mathrm{FDG}$ PET/ CT images (a coronal slice; $\mathbf{b}$ sagittal slice; $\mathbf{c}$ axial slice) of a patient with a
PVE showing two foci of FDG uptake in the prosthetic mitral valve (red arrows, SUVmax: 4.4; purple arrows, SUVmax 3.5) 


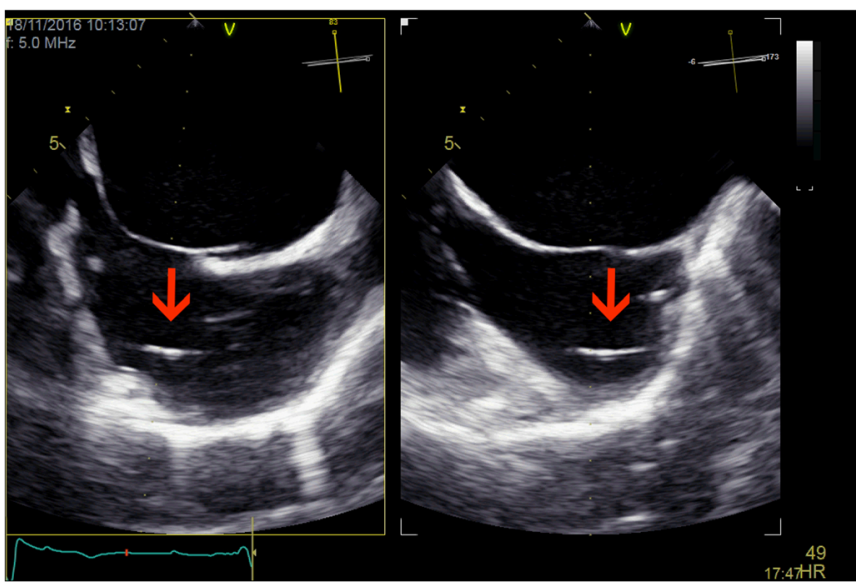

Fig. 3 To the left of the picture, transoesophageal ultrasound of a patient with a pacemaker suffering from a fever of undetermined origin; absence of obvious lesion identified. To the right of the picture, $\left[{ }^{18} \mathrm{~F}\right] \mathrm{FDG}$ PET/CT images (a maximum intensity projection of PET images; $\mathbf{b}, \mathbf{c}$ axial slices)

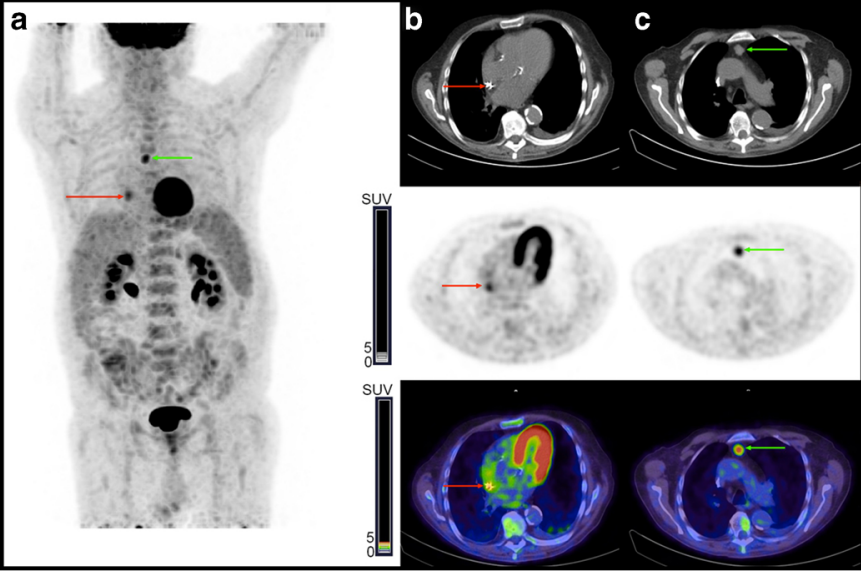

of a patient with a CIED. Images show a focus of FDG uptake in pacemaker leads in the superior vena cava (a, b red arrows, SUVmax 4.7) and a FDG avid reactive lymph node in the mediastinum (a, c green arrows, SUVmax 5.4) limited to the pocket or leads from a more severe infection affecting the whole device [23] (Table 2). Therefore, both imaging approaches can be of help in patients with suspected CDRIE (ESC class Illb recommendations) [5].

\section{Detection of Metastatic Infectious Events}

Embolic events are a frequent and life-threatening complication of IE. They occur in 20-50\% of patients and can be totally silent in $20-50 \%$ of cases. The brain and spleen are the most affected in the left IE while the lungs complicate the right IE, particularly in CDRIE [5]. In the recent ESC guidelines, the imaging detection of septic emboli clearly impacts on the Duke score and consequently on the diagnostic certainty of IE (minor criterion) and decision-making. In fact, as reported by several authors, $\left[{ }^{18} \mathrm{~F}\right]$ FDG PET/CT is capable of detecting distant embolic sites $(15 \%)$ with a reasonable sensitivity (14-100\%) and specificity (80\%), most of which clinically silent (up to $30 \%$ ), and previously undiagnosed tumours $(6.5 \%)$, many of them in early stages and potentially curable $[12,24]$. This approach is relatively costeffective and may avoid using additional ionizing radiologic techniques. $\left[{ }^{18} \mathrm{~F}\right] \mathrm{FDG}$ PET/CT is very accurate in organs with low physiological uptake, therefore not applicable in ruling out the presence of brain embolism [25•]. Radiolabelled leukocytes SPECT/CT shares with PET/CT the possibility of acquiring whole-body images and by performing additional planar and SPECT/CT spot images also constitutes an invaluable aid for detecting septic embolism even in asymptomatic patients [14, $23 \bullet$.

\section{Left Ventricular Assist Device Associated Infections}

The prevalence of infection and sepsis in left ventricular assist device (LVAD) patients ranges from 23 to $58 \%$, being associated with a high mortality rate (15-44\%) [26]. The major sites of infection comprise the mediastinum drivelines and device surface, so called « LVAD endocarditis » [27]. In a recent retrospective study concerning 31 patients, $\left[{ }^{18} \mathrm{~F}\right] \mathrm{FDG}$ PET/CT yielded a sensitivity of $100 \%$ and a specificity of $94 \%$ for the diagnosis of LVAD infection [28]. Litzler et al. also highlighted the potential usefulness of radiolabelled leukocytes SPECT/CT in the management of LVAD infection (8 patients) by determining the precise anatomic location and extent of a suspected infection [26]. The evidence in this setting is, however, still quite limited.

\section{Limitations and Technical Considerations}

Despite significant advantages, molecular imaging techniques still present limitations that would require further developments (Table 1). The majority of them are related to the complexity of imaging interpretation, particularly when $\left[{ }^{18} \mathrm{~F}\right] \mathrm{FDG}$ is used. In fact, several pathological conditions can mimick the pattern of focally increased uptake such as non-infected tissue (active thrombi, atherosclerotic plaques, vasculitis, tumours, lipomatous hypertrophy of the inter-atrial septum, foreign body reactions (i.e. BioGlue surgical adhesive used to seal the aortic graft at time of surgery)). Timing of imaging in relation to surgical intervention still remains an open issue since up to 4-8 weeks, the persistence of post-operative inflammatory response with false positive results is possible. Clear definition of the impact of antimicrobial treatment (risk of false negative cases) as well as of blurring artefacts has also to be established. In addition, the problem of false negative results in presence of small oscillating vegetation and metastatic foci $<5 \mathrm{~mm}$ which are below the threshold of detectability/resolution has to be considered as well as the issue of radiation burden that might increase when certain protocol for imaging acquisition are applied (i.e. retrospective ECG-triggering) $[6,29]$.

The best strategy to reduce false positive finding is correct patient preparation and selection. We want to underline, once 
Fig. 4 Proposed algorithm incorporating nuclear imaging and current guidelines in diagnosis IE $[1,6 \bullet \bullet]$

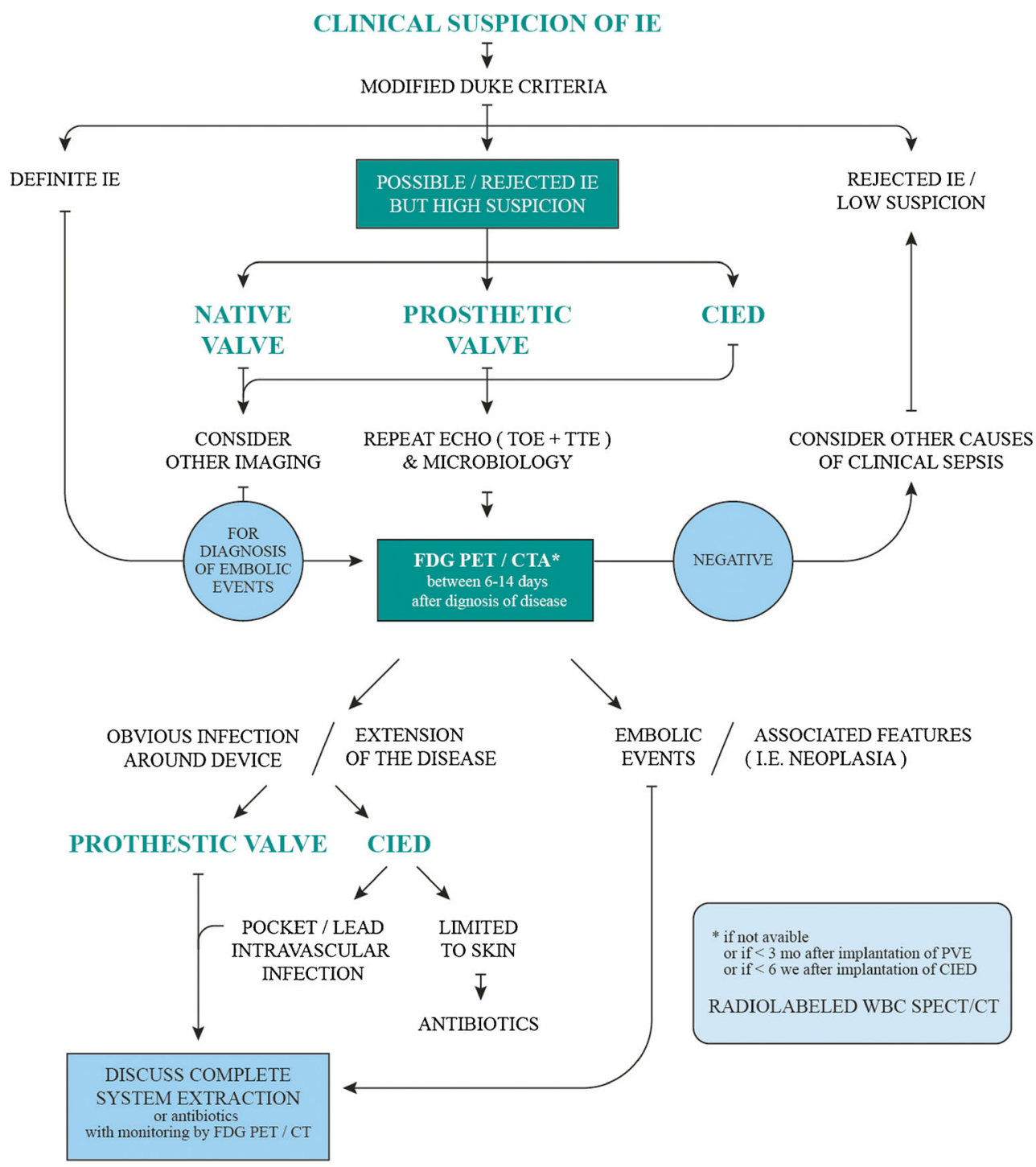

more, how important is the optimal suppression of physiologic myocardial glucose utilization with (1) high fat, low carbohydrate diet for at least two meals with a fast of at least $4 \mathrm{~h}$ prior imaging [24, 30•]; (2) possible use of intravenous unfractionated heparin $(50 \mathrm{IU} / \mathrm{kg})$ approximately $15 \mathrm{~min}$ prior to ${ }^{18} \mathrm{~F}$-FDG injection $[16,31]$. In addition, we also recommend to withdraw of metformin which is associated with intense and diffuse $\left[{ }^{18} \mathrm{~F}\right] \mathrm{FDG}$ uptake in the small and large bowels [32•]; (3) discontinuation/reduction to the lowest possible dose the use of steroid in the $24 \mathrm{~h}$ preceding the exam [32•]; (4) avoid the inclusion of patients $<3$ months of PV implantation [5].

Of note, neither diabetes nor hyperglycemia at the time of the study seems to increase the false-negative rate; a blood glucose level below $200 \mathrm{mg} / \mathrm{dL}$ might be suggested [32]. At this stage, there is no evidence to routinely recommend antimicrobial treatment discontinuation before performing $\left[{ }^{18} \mathrm{~F}\right] \mathrm{FDG}$ PET/CT. However, the use of antimicrobial should be taken into account during imaging interpretation since they might affect the intensity of FDG uptake due to their immunomodulatory effect [7]. Late PET/CT imaging (2-3 h after FDG injection) should be considered in patients when negative 1-h PET/Ct scan in maximizing the contrast between septic foci and background [33].

As compared to $\left[{ }^{18} \mathrm{~F}\right] \mathrm{FDG} \mathrm{PET} / \mathrm{CT}$, radiolabelled leukocyte imaging is more time consuming, necessitates in-house leukocytes labelling with direct handling of blood products, requiring specific experience (and should be performed in trained centres) $[19,32 \bullet, 34 \bullet]$. As for $\left[{ }^{18} \mathrm{~F}\right] \mathrm{FDG}$ PET, the major goals of radiolabelled leukocytes SPECT/CT are to minimize tracer uptake in normal tissues, while maintaining uptake in target tissues. False positive findings have been described in distant septic embolisms (cold spot in spleen/kidney embolism or spondylodiskitis; hot spot in benign or malign process) but not in cardiac infection [14]. False negative scans have been observed (1) in patients with small valve vegetation $(<6 \mathrm{~mm}$, limit of spatial resolution), (2) in case of IE by some strains 
(Staphylococcus epidermidis, Enterococcus, Candida, Coxiella burnetii) able to form a biofilm limiting neutrophil recruitment at the primary site of IE and the local efficacy of antimicrobial treatment, (3) in case of drained abscess [14, 32•]. Of note, the impact of antibiotic therapy has not been specifically examined.

\section{Perspectives and Future Developments}

The ideal molecular imaging modality would allow for a reliable detection of infection at an early stage, differentiate infection from other causes of inflammation, provide species (causative micro-organism) and resistance information to guide optimal therapy and monitor disease progression, contribute to shorten the hospital stay, prevent clinical complications, and reduce the cost of hospitalization. Such an imaging modality would require discovery of pathogenic mechanisms that can serve as imaging targets (i.e. fibrin, platelet, granulocytes, etc.), development and validation of new pathogen-specific probes (bacteria-targeted imaging approaches), and implementation of technologies that are capable of specific IE detection (targeted molecular imaging), combine molecular imaging to anatomical imaging with high soft-tissue contrast such as PET/ MRI (magnetic resonance imaging) and/or providing detectors with higher sensitivity and spatial resolution (i.e. digital PET/ CT and cadium-zinc-telluride camera) $[35,36]$.

\section{Conclusion}

Functional nuclear imaging has gained growing interest in the diagnosis and management of IE (Fig. 4). Radiolabelled leukocyte SPECT/CT seems to be more specific for the detection of IE and infectious foci than ${ }^{18}$ F-FDG $\left[{ }^{18} \mathrm{~F}\right]$ FDG PET/CT. Nevertheless, $\left[{ }^{18} \mathrm{~F}\right]$ FDG $\mathrm{PET} / \mathrm{CT}$ is likely the preferred first-line metabolic imaging technique since SPECT/CT is less sensitive, more time consuming and require in-house leukocyte labelling. Radiolabelled leukocytes could be reserved for doubtful cases with PET/CT. Nonetheless, radiolabelled leukocytes could be particularly useful in early post-operative period and in NVE. Further research should focus on defining the best protocol and the ideal timing for image acquisition and on the development of more specific probes.

\section{Compliance with Ethics Guidelines}

Conflict of Interest Paola Anna Erba reports grants, personal fees and non-financial support from AAA, grants and non-financial support from Sigma Tau, personal fees from Springer, non-financial support from Gammaservizi, and personal fees and non-financial support from GE Healthcare, outside the submitted work.

All other authors declare that they have no conflict of interest.
Human and Animal Rights and Informed Consent This article does not contain any studies with human or animal subjects performed by any of the authors.

\section{References}

Paper of particular interest, published recently, have been highlighted as:

- Of importance

•- Of major importance

1.• Habib G, Lancellotti P, Antunes MJ, Bongiorni MG, Casalta JP, Del Zotti JP, et al. ESC guidelines for the Management of Infective Endocarditis: the task force for the management of infective endocarditis of European Society of Cardiology (ESC). Endorsed by: European Association for Cardio-Thoraric Surgery (EACTS), the European Association of Nuclear Medecine (EANM). Eur Heart J. 2015;36: $3075-123$. There are the first guidelines on the purpose that integrate the role of nuclear imaging in infective endocarditis.

2. Li JS, Sexton DJ, Mick N, Nettles R, Fowler VG Jr, Ryan T, et al. Proposed modifications to the Duke criteria for the diagnosis of infective endocarditis. Clin Infect Dis. 2000;30:633-8.

3. Thuny F, Grisoli D, Collart F, Habib G, Raoult D. Management of infective endocarditis: challenges and perspectives. Lancet. 2012;379(9819):965-75.

4.• Lancellotti P, Habib G, Oury C, Nchimi A. Positron emission tomography/computed tomography imaging in device infective endocarditis ready for prime time. Circulation. 2015;132:1076-80. This editorial provides a recent update on the usefulness of nuclear imaging on CIED infections.

5. Ricciardi A, Sordillo P, Ceccarelli L, Maffongelli G, Calisti G, Di Pietro B, et al. 18-Fluoro-2-deoxyglucose positron emission tomography-computed tomography: an additional tool in the diagnosis of prosthetic valve endocarditis. Int J Infect Dis. 2014:219-24.

6.• Sarrazin JF, Philippon F, Trottier M, Tessier M. Role of radionuclide imaging for diagnosis of device and prosthetic valve infections. World J Cardiol. 2016;8(9):534 46. This study is a recent review of ${ }^{18}$ F-FDG PET/CT and WBC SPECT/CT in clinical practice in patients with CIED infection and PVE.

7. Millar BC, Prendergast BD, Alavi A, Moore JE. ${ }^{18}$ FDG-positron emission tomography (PET) has a role to play in the diagnosis and therapy of infective endocarditis and cardiac device infection. Int $\mathbf{J}$ Cardiol. 2013;167:1724-36.

8. Chen W, Kim J, Molchanova-Cook OP, Dilsizian V. The potential of FDG PET/CT for early diagnosis of cardiac and prosthetic valve infection before morphologic Damges ensue. Cuur Cariol Rep. 2014;16:459. FDG-PET/CT makes it possible to distinguish different types of CIED's infections involving different management. This study also emphasizes the importance of patient preparation.

9. Dorbala S, Di Carli MF, Delbeke D, Abbara S, DePuey EG, Dilsizian V, et al. SNMMI/ASNC/SCCT guideline for cardiac SPECT/CT and PET/CT 1.0. J Nucl Med. 2013;54:1485-507.

10. Musso M, Petrosillo N. Nuclear medicine in diagnosis of prosthetic valve endocarditis: an update. Biomed Res Int. 2015;127325. Review of the literature to assess the available evidence on the role of nuclear medecine techniques in the diagnosis of PVE.

11.• Kouijzer IJ, Vos FJ, Janssen MJR, van Dijk APJ, Oyen WJG, BleekerRovers CP. The value of 18F-FDG PET/CT in diagnosing infectious endocarditis. Eur J Nucl Med Mol Imaging. 2013;40:1102-7. There is the first large series of prospectively included patients investigating the value of ${ }^{18}$ F-FDG PET/CT in definite infectious endocarditis. 
12.• Gomes A, Glaudemans AW, Touw DJ, Van Melle JP, Willems TP, Maass AH, et al. Diagnostic value of imaging in infective endocarditis: a systematic review. Lancet Infect Dis. 2017;17:e1-e14. This article reviews the literature with regard to the potential value of ${ }^{18}$ F-FDG PET/CT, WBC SPECT/CT and ECG-gated multidetector $\mathrm{Ct}$ angiography on infective endocarditis as well as the pitfalls and limitations of these technical modalities.

13. Vos F, Bleeker-Rovers CP, Kullberg BJ, Adang EM, Oyen WJ. Cost-effectiveness of routine ${ }^{18} \mathrm{~F}$-FDG PET/CT in high-risk patients with gram-positive bacteremia. J Nucl Med. 2011;52:16738 . The introduction in routine of $18 \mathrm{~F}$-FDG PET/CT decrease the cost by the early diagnosis of precocious metastatic infectious foci.

14.• Erba PA, Conti U, Lazzeri E, Sollini M, Doria R, De Tommasi SM, et al. Added value of ${ }^{99 \mathrm{~m}} \mathrm{Tc}-\mathrm{HMPAO}-$ labelled leukocyte SPECT/ $\mathrm{CT}$ in the characterization and management of patients with infectious endocarditis. J Nucl Med. 2012;53:1235-43. This is the largest study demonstrating the interest of the WBC SPECT/CT in diagnosis of infective endocarditis.

15. Baddour LM, Wilson WR, Bayer AS, Fowler VG Jr, Bolger AF, Levison ME, et al. Infective endocarditis : diagnosis, antimicrobial therapy, and manahement of complications: a statement for healthcare professionals from the committee on rheumatic fever, endocarditis, and Kawasaki disease, council on cardiovascular disease in the young, and the councils on clinical Cardiology, stroke, and cardiovascular surgery and anesthesia; American Heart Association: endorsed by the Infectious Diseases Society of America. Circulation. 2005;111:e394-434.

16. Tanis W, Budde RPJ, Van der Bilt IAC, Delemarre B, Hoohenkerk G, Van Rooden JK, et al. Novel imaging stratregies for the detection of prosthetic heart valve obstruction and endocarditis. Neth Heart J. 2016;24:96-107.

17.• Saby L, Laas O, Habib G, Cammilleri S, Mancini J, Tessonnier L, et al. Positron emission tomography/computed tomography for diagnosis of prosthetic valve endocarditis: increased valvular $18 \mathrm{~F}$ fluorodeoxyglucose uptake as a novel major criterion. J Am Coll Cardiol. 2013;61:2374-82. This propective study shows that adding abnormal FDG uptake around the prosthetic valve is a new major criterion that increased the sensitivity of the modified Duke criteria.

18.• Pizzi MN, Roque A, Fernández-Hidalgo N, Cuéllar-Calabria H, Ferreira-González I, Gonzàlez-Alujas MT, et al. Improving the diagnosis of infective endocarditis in prosthetic valves and Intracardiac devices with $18 \mathrm{~F}$-Fluordeoxyglucose positron emission tomography/computed tomography angiography: initial results at an infective endocarditis referral center. Circulation. 2015;132: 1113-26. The interest of PET/CTA is clearly demonstrated in diagnosis of infective endocarditis.

19. Hyafil F, Rouzet F, Lepage L, Benali K, Raffoul R, Duval X, et al. Role of radiolabelled leucocyte scintigraphy in patients with a suspicion of prosthetic valve endocarditis and inconclusive echocardiography. Eur Heart J Cardiovasc Imaging. 2013;14:586-94. Study who demonstrates excellent positive predictive value of SPECT/ CT to detection of perivalvular infection and abscesses in patients with suspicion of PVE.

20. Ploux S, Riviere A, Amroui S, et al. Positron émission tomography in patients with suspected pacing system infections may play a critical role in difficul cases. Heart Rythm. 2011;8:1478-81.

21. Sarrazin JF, Philippon F, Tessier M, et al. Usefulness of fluorine-18 positron émission tomography/computed tomography for identification of cardiovascular implantable electronic device infections. J Am Coll Cardiol. 2012;59:1616-25. FDG-PET/CT is useful in differentiating between CIED infection and recent post-implant change.

22.• Granados U, Fuster D, Pericas JM, Llopis JL, Ninot S, Quintana E, et al. Diagnostic accuracy of ${ }^{18} \mathrm{~F}-\mathrm{FDG}$ PET/CT in infective endocarditis and implantable cardiac electronic device infection: a cross-sectional study. J Nucl Med. 2016;57:1726-32. It is a considerable prospective study demonstrating the interest of ${ }^{18} \mathrm{~F}$ FDG PET/CT in CIED infections.

23. Erba PA, Sollini M, Conti U, Bandera F, Tascini C, De Tommasi S, et al. Radiolabelled WBC scintigraphy in the diagnostic workup of patients with suspected device-related infections. JACC Cardiovasc Imaging. 2013;6:1075-86. Interest of 99m Tc-HMPAO-WBC to diagnose CIED infection and detection of associated complication.

24. Van Riet J, Hill EE, Gheysens O, Dymarkowski S, Herregods MC, Herijgers P, et al. ${ }^{18} \mathrm{~F}$-FDG PET/CT for early detection of embolism with infective endocarditis. Eur j Nucl Mol Imaging. 2010;37:1189-97.

25. Ozcan C. Asmar a, gill S, Thomassen a and Diederichsen ACP. The value of FDG-PET/CT in the diagnostic work-up of extracardiac infectious manifestations in infectious endocarditis. Int $\mathrm{J}$ Cardiovasc Imaging. 2013;29:1629-37. Role of FDG-PET/CT in the assessment of extra-cardiac complications.

26. Litzler PY, Manrique A, Etienne M, Salles A, Eded-Sanson A, Bessou JP, et al. Leukocyte SPECT/CT for detecting infection of left-ventricular-assist devices: preliminary results. J Nucl Med. 2010;51:1044-8.

27. Wickline SA, Fischer KC. Can infections be imaged in implanted devices? ASAIO J. 2000;46:S80-1.

28. Dell'Aquila AM, Mastrobuoni S, Alles S, Wenning C, Henryk W, Schneider SR, et al. Contributory role of fluorine 18 Fluorodeoxyglucose positron emission tomography/computed tomography in the diagnosis and clinical Management of Infections in patients supported with a continuous-flow left ventricular assist device. Ann Thorac Surg. 2016;101:87-94.

29. Scholtens AM, Swart LE, Verberne HJ, Tanis W, Lam MG, Budde RP. Confounders in FDG-PET/CT imaging of suspected prosthetic valve endocarditis. JACC Cardiovasc Imaging. 2016;9:1462-5.

30. Orvin K, Goldberg E, Bernstine H, Groshar D, Sagie A, Kornowski $\mathrm{R}$, et al. The role of FDG-PET/CT imaging in early detection of extra-cardiac complications of infective endocarditis. Clin Microbiol Infect. 2015;21:69-76. Role of FDG-PET/CT to highlight extra-cardiac complications, prospective study.

31. Tlili G, Amroui S, Mesguich C, Rivière A, Bordachar P, Hindié E. High Perfomances of ${ }^{18} \mathrm{~F}$-fluorodeoxyglucose PET-Ct in cardiac implantable device infections: a study of 40 patients. J Nucl Cardiol. 2015;22:787-98.

32. Glaudemans AWJM, Israel O, RHJA S. Pitfalls and limitations of radionuclide and hybrid imaging in infection and inflammation. Semin Nucl Med. 2015;45:500-12. Focus on general pitfalls that can occur in infection and inflammation imaging, in particular, FDG-PET/CT and SPECT/CT.

33. Leccisotti L, Perna F, Lago M, Leo M, Stefanelli A, Calcagni M, et al. Cardiovascular implantable electronic device infection: delayed vs standard FDG PET-CT imaging. J Nucl Cardiol. 2014:21:622-32.

34. Rouzet F, Chequer R, Benali K, Lepage L, Ghodbane W, Duval X, et al. Respective performance of ${ }^{18} \mathrm{~F}$-FDG PET/CT and Radiolabelled leukocyte Scintography for the diagnosis of prosthetic valve endocarditis. J Nucl Med. 2014;55:1-6. Study comparing the efficiency between PET/CT and SPECT/CT.

35. Gratz S, Raddatz D, Hagenah G, Behr T, Becker W. ${ }^{99 \mathrm{~m}}$ TC-labelled antigranulocyte monoclonal antibody FAB' fragments versus echocardiography in the diagnosis of subacute infective endocarditis. Int J Cardiol. 2000;75:75-84.

36. Caobelli F, Wollenweber T, Bavendiek U, Khün C, Schütze C, Geworski K, et al. Simultaneous dual-isotope solid-state detector SPECT for improved tracking of white blood cells in suspected endocarditis. Eur Heart J. 2016;28. pii: ehw:231. 